\title{
Air quality in street canyons: a case study
}

\author{
F. Patania ${ }^{1}$, A. Gagliano ${ }^{1}$, F. Nocera ${ }^{2}$ \& A. Galesi ${ }^{1}$ \\ ${ }^{I}$ Energy and Environment Division of D.I.I.M., \\ Engineering Faculty of University of Catania, Italy \\ ${ }^{2}$ Department of Analysis, Representation and \\ Project in Mediterranean Areas, \\ Architectural Faculty of University of Catania, Italy
}

\begin{abstract}
Keeping the air quality acceptable has become an important task for decision makers as well as for non-governmental organizations. Particulate and gaseous emissions of pollutants from auto-exhausts are responsible for rising discomfort, increasing airway diseases, decreasing productivity and the deterioration of artistic and cultural patrimony in urban centers. Air quality limit values, which are aimed at protecting public health, are frequently exceeded especially in streets and other urban hotspots. Within these streets, pedestrians, cyclists, drivers and residents are likely to be exposed to pollutant concentrations exceeding current air quality standards.

In order to give the right support to decision makers for air pollution control, a suitable microscale dispersion model must be used to investigate phenomenon The paper presents the results obtained by utilizing a three dimensional numerical model based on Reynolds-averaged Navier-Stokes equations to simulate the fluid-flow development and pollutant dispersion within an isolated street canyon. Finally, the authors tested the reliability of the same code examined resemblances and differences between the measured data coming from a survey measurement within the canyon and the data coming from the code.
\end{abstract}

Keywords: urban canyon, air pollution, traffic emissions, CFD.

\section{Introduction}

Despite significant improvements in fuel and engine technology, urban environments are mostly dominated by traffic emissions. Most of the substances 
directly emitted by vehicles in the atmospheric or indirectly produced through photochemical reactions could represent a serious hazard for human health.

In urban environments and especially in those areas where population and traffic density are relatively high, human exposure to hazardous substances is expected to be significantly increased [1]. This is often the case near busy traffic axis in city centres where urban topography and microclimate may contribute to the condition of air stagnation rising the contamination hotspots. High pollution levels have been observed in "Urban Canyons", which is a term frequently used for urban streets bounded by buildings on both sides [2].

The dimensions of an urban canyon are usually expressed by its aspect ratio $[3,4]$, which is the height $(\mathrm{H})$ of the canyon divided by the width $(\mathrm{W})$. A canyon might be called regular, if it has an aspect ratio approximately equal to 1 and not big openings along the walls. An avenue canyon may have an aspect ratio below 0.5 , while a value of 2 may be representative of a deep canyon. Finally, the length (L) of the canyon usually expresses the road distance between two major intersections, subdividing street canyons into short $(\mathrm{L} / \mathrm{H} \approx 3)$, medium $(\mathrm{L} / \mathrm{H} \approx 5)$; and long canyons $(\mathrm{L} / \mathrm{H} \approx 7)$. Urban Canyons might be also classified in symmetric canyons if the buildings bounding the street have approximately the same height. On the contrary they may be classified asymmetric, if there are significant differences in the building heights forming the canyon.

The study on wind flow and pollutant transport inside and over urban street canyons, have attracted great concern during the past two decades mainly due to increasing urban pollutants and their adverse impacts on human health.

Field measurements [5], laboratory-scale physical modelling [6] and computational fluid dynamics (CFD) techniques [7] are the common tools used to study the wind flow and pollutant distributions in street canyons.

A three-dimensional numerical model based on Reynolds-averaged NavierStokes equations coupled $\mathrm{k}-\varepsilon$ turbulence models was developed to simulate the fluid-flow development and pollutant dispersion within an isolated street canyon using the FLUENT code.

The field experiment used to validate the model outputs is described in Section 2 followed by the model descriptions and results in Section 3 and 4. The flow and concentrations predicted by the FLUENT code are then compared with the measurements from the field experiment in Section 4 along with a discussion of the discrepancies.

\section{Experiment}

\subsection{Canyon geometry and sampling methods}

The Vincenzo Giuffrida Street canyon (Fig. 1) is located in a residential part of Catania city. Regular net of perpendicularly intersecting street canyons forms this urban area. Information about geometry of the urban canyon was obtained from Catania Council

The studied canyon has a NNW orientation and its main axis is 345 degrees from the North. The canyon is $250 \mathrm{~m}$ length, $10 \mathrm{~m}$ width and the average height 
of the buildings is $21 \mathrm{~m}$. The Balconies facades were taken into account in the model because they significantly influence airflow in the façade boundary layer. The authors, also, considered the roof of the buildings flat.

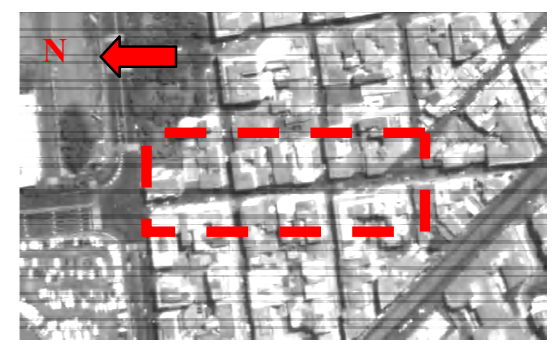

Figure 1: $\quad$ Area of the case study.

The canyon is modelled without trees and parked cars. Undisturbed wind velocity and wind directions were obtained by an anemometer located on the rooftop at a height of $25 \mathrm{~m}$ above ground level. Measured wind conditions were used as a parameter input for numerical predictions. A portable anemometer Testo 452, located on 1th floor, was used to compare predicted and measured values.

The wind and turbulence measurements were averaged over a period of 15 minutes.

Carbon monoxide measurements were taken in the street using $\mathrm{CO}$ monitor based on the non-dispersive infrared method (NDIR). The carbon monoxide, in street canyons, is primarily produced by petrol engines and it is practically inert on short timescales. It is usually considered as a gas tracer of the dispersion of traffic-related pollutants.

\subsection{Traffic flow measurement}

The measurements recorded were 15-min average of 1-min sampled.

Traffic data was registered using a video camera. A video camera was mounted at the roadside near the north entrance of the street to record the flow of traffic during the rush hours (7:30-8:30, 10:00-11:00, 13:00-14:00 and 18:0019:00). The tape was then replayed on the laboratory television to count the number of each vehicles. Vehicles were classified as vehicles (V), heavy vehicles (HV) and motorcycles (MC).

\section{Model description}

\subsection{Governing equation}

The numerical model used in this study is Airpak which is a three-dimensional, steady, $\mathrm{k}-\varepsilon$ turbulence model applied to investigate the high Reynolds number skimming flow field of an urban canyon. The choice comes from the capability of Airpak to deal with complex shaped walls and other boundary conditions 
using flexible fine-scale grids. Airpak is a CFD modelling based on the numerical solution of the governing fluid flow and dispersion equations which are derived from basic conservation and transport principles: the mass conservation (continuity) equation, the three momentum conservation (NavierStokes) equations in $\mathrm{x} ; \mathrm{y} ; \mathrm{z}$; the transport equation for pollutant concentration. The equations of state (obtained through the thermodynamic equilibrium assumption) and the Newtonian model of viscous stresses are also enlisted to close the system numerically, while initial and boundary conditions have to be specified to characterize external wind flows by the wind direction, wind speed, its height above the ground, and description of the local terrain.

The code solves the Navier-Stokes equations for transport of mass, momentum, species, and energy when it calculates laminar flow with heat transfer. Additional transport equations are solved when the flow is turbulent or when radioactive heat transfer is included.

The equation for conservation of mass, or continuity equation, can be written as:

$$
\frac{\partial \rho}{\partial t}+\frac{\partial \rho}{\partial x_{i}}\left(\rho \cdot u_{i}\right)=0
$$

Transport of momentum in the $\mathrm{i}^{\text {th }}$ direction in an inertial (non-accelerating) reference frame is described by

$$
\frac{\partial}{\partial t}\left(\rho \cdot u_{i}\right)+\frac{\partial}{\partial x_{j}}\left(\rho \cdot u_{i} \cdot u_{j}\right)=\frac{\partial p}{\partial x_{i}}+\frac{\partial \tau_{i j}}{\partial x_{j}}+\rho \cdot g_{i}+F_{i}
$$

where $\mathrm{p}$ is the static pressure, $\tau_{\mathrm{ij}}$ is the stress tensor, and $\rho \mathrm{g}_{\mathrm{i}}$ is the gravitational body force in the i direction. $\mathrm{F}_{\mathrm{i}}$ contains other source terms that may arise from resistances, sources, etc. The energy conservation equation for a fluid region written in terms of sensible enthalpy $\mathrm{h}$ is:

$$
\frac{\partial}{\partial t}(\rho \cdot h)+\frac{\partial}{\partial x_{i}}\left(\rho \cdot u_{i} \cdot h\right)=\frac{\partial}{\partial x_{i}}\left(k+k_{t}\right) \cdot \frac{\partial T}{\partial x_{i}}+S_{h}
$$

where $\mathrm{k}$ is the molecular conductivity, $\mathrm{k}_{\mathrm{t}}$ is the conductivity due to turbulent transport and the source term $S_{h}$ includes any volumetric heat sources you have defined. The species transport equations takes the following general form

$$
\frac{\partial}{\partial t}\left(\rho \cdot m_{i^{\prime}}\right)+\frac{\partial}{\partial x_{i}}\left(\rho \cdot u_{i} \cdot m_{i^{\prime}}\right)=-\frac{\partial}{\partial x_{i}} J_{i^{\prime} i}+S_{i^{\prime}}
$$

where $\mathrm{Si}^{\prime}$ is the rate of creation by addition from user-defined sources.

The code used the standard k- $\varepsilon$ model based on model transport equations for the turbulent kinetic energy $(\mathrm{k})$ and its dissipation rate $(\varepsilon)$. The model transport equation for $\mathrm{k}$ is derived from the exact equation, while the model transport equation for $\varepsilon$ is obtained using physical reasoning and bears little resemblance to its mathematically exact counterpart. The turbulent kinetic energy, $k$, and its rate of dissipation, $\varepsilon$, are obtained from the following transport equations:

$$
\rho \frac{\mathrm{Dk}}{\mathrm{Dt}}=\frac{\partial}{\partial \mathrm{x}_{\mathrm{i}}}\left[\left(\mu+\frac{\mu_{\mathrm{t}}}{\sigma_{\mathrm{k}}}\right) \frac{\partial \mathrm{k}}{\partial \mathrm{x}_{\mathrm{i}}}\right]+\mathrm{G}_{\mathrm{k}}+\mathrm{G}_{\mathrm{b}}-\rho \varepsilon
$$




$$
\rho \frac{\mathrm{D} \varepsilon}{\mathrm{Dt}}=\frac{\partial}{\partial \mathrm{x}_{\mathrm{i}}}\left[\left(\mu+\frac{\mu_{\mathrm{t}}}{\sigma_{\varepsilon}}\right) \frac{\partial \varepsilon}{\partial \mathrm{x}_{\mathrm{i}}}\right]+\mathrm{C}_{1 \varepsilon} \cdot \frac{\varepsilon}{\mathrm{k}} \cdot\left(\mathrm{G}_{\mathrm{k}}+\mathrm{C}_{3 \varepsilon} \mathrm{G}_{\mathrm{b}}\right)-\mathrm{C}_{2 \varepsilon} \rho \frac{\varepsilon^{2}}{\mathrm{k}}
$$

In these equations, $G_{k}$ represents the generation of turbulent kinetic energy due to the mean velocity gradients, $G_{b}$ is the generation of turbulent kinetic energy due to buoyancy, $\mathrm{C}_{1 \varepsilon}, \mathrm{C}_{2 \varepsilon}$, and, $\mathrm{C}_{3 \varepsilon}$ are constants. $\sigma_{\mathrm{k}}$ and $\sigma_{\varepsilon}$ are the turbulent Prandtl numbers for $k$ and $\varepsilon$ respectively The "eddy" or turbulent viscosity, $\mu_{t}$, is computed by combining $\mathrm{k}$ and $\varepsilon$.

$$
\mu_{\mathrm{t}}=\rho \mathrm{C}_{\mu} \frac{\mathrm{k}^{2}}{\varepsilon}
$$

The model constants have the following default values $\mathrm{C}_{1 \varepsilon}=1,44, \mathrm{C}_{2 \varepsilon}=1.92$; $\mathrm{C}_{\mu}=0.09 \sigma_{\varepsilon}=1.3$.and $\sigma_{\mathrm{k}}=1.0$. The degree to which $\varepsilon$ is affected by the buoyancy is determined by the constant $\mathrm{C}_{3 \varepsilon}$

$$
\mathrm{C}_{3 \varepsilon}=\tanh \left|\frac{\mathrm{v}}{\mathrm{u}}\right|
$$

where $\mathrm{v}$ is the component of the flow velocity parallel to the gravitational vector and $\mathrm{u}$ is the component of the flow velocity perpendicular to the gravitational vector. In this way, $\mathrm{C}_{3 \varepsilon}$ will become 1 for buoyant shear layers for which the main flow direction is aligned with the direction of gravity. For buoyant shear layers that are perpendicular to the gravitational vector, $\mathrm{C}_{3 \varepsilon}$ will become zero.

\subsection{Estimation of pollutant emission rate by traffic}

The rate of emission $\mathrm{q}_{\mathrm{ik}}$ by traffic in the $\mathrm{k}_{\mathrm{th}}$ lane, of species $\mathrm{i}$, is given by:

$$
q_{i k}(t)=\frac{E F_{i k}(t) \cdot N_{k}(t)}{A_{k} \cdot 1000}
$$

where $\mathrm{EF}_{\mathrm{ik}}$ is the emission factor of pollutant $\mathrm{i}$, and $\mathrm{N}_{\mathrm{k}}$ is the average traffic flow rate (or number of vehicles per unit time); the subscript $\mathrm{k}$ refers to the $\mathrm{k}^{\text {th }}$ lane. $\mathrm{N}_{\mathrm{k}}$ is determined from measurements (see figure 2), so $\mathrm{q}_{\mathrm{ik}}$ can be evaluated once $\mathrm{EF}_{\mathrm{ik}}$ is known utilizing COPERT III methodology [8, 9].

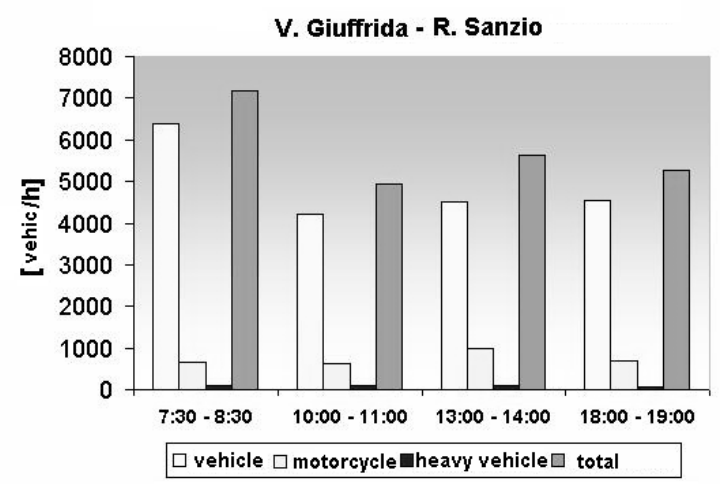

Figure 2: Traffic flow. 
$A_{k}\left(=h_{k} W_{k}\right)$ in the above equation is the cross-sectional area of the $k^{\text {th }}$ line source, where $h_{k}$ and $w_{k}$ are the height and bandwidth of the line source. The typical values of $\mathrm{h}_{1}=1.2 \mathrm{~m}$ and $\mathrm{w}_{1}=1.9 \mathrm{~m}$ for a passenger car.

Finally the continuous linear source, typical of vehicular emission in stationary condition, was schematized as twenty-eight lined up point sources $(0,20 \mathrm{~m}$ above the ground) uniformly distributed along the axis of the canyon.

The traffic emissions on the streets outside the street canyon were not considered.

\subsection{Model domain and boundary conditions}

As shown in Fig. 7 the chosen domain of calculus, in Cartesian coordinates, has the following dimensions: $x=700 \mathrm{~m}, \mathrm{y}=100 \mathrm{~m}$ and $\mathrm{z}=700 \mathrm{~m}$

In modelling urban flow and dispersion, smaller grid sizes would be better located near buildings to solve flow and dispersion fields, but away from buildings larger grid sizes are allowed. To make the CFD model efficient for a given computing resource, a non-uniform grid system is implemented in the model.
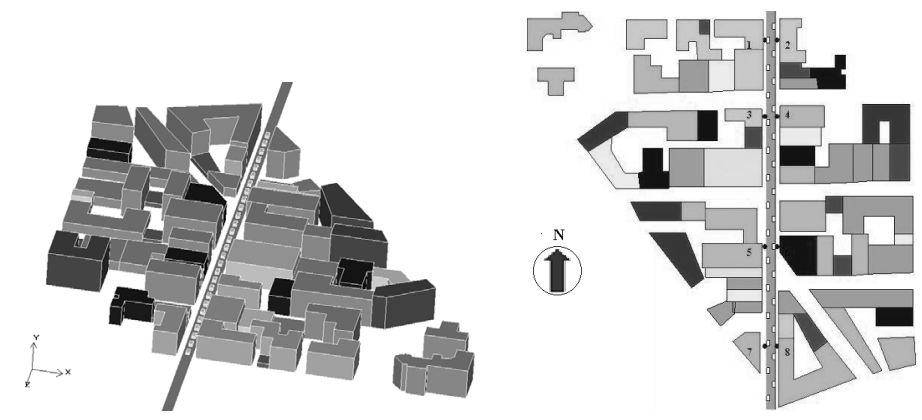

Figure 3: Buildings in 3D Domain (on the right) and plan of the case studied (on the left).

The grid of calculus becomes thicker and thicker inside the urban canyon and close to the ground level. The maximum dimension of the grid is $x=25 \mathrm{~m}, \mathrm{y}=10$ $\mathrm{m}$ and $\mathrm{z}=25 \mathrm{~m}$ on the Atmospheric Boundary Layer (ABL), while the minimum dimension of the mesh is $\mathrm{x}=\mathrm{y}=\mathrm{z}=0,01 \mathrm{~m}$ on the exhausts pipe. Accurate simulation of ABL flow in the computational domain is imperative to obtain accurate and reliable predictions of the related atmospheric processes.

The external wind flows was characterized by the wind direction, wind speed, its height above the ground, and a description of the local terrain using the following equations:

$$
\mathrm{U}(\mathrm{h})=\left\{\begin{array}{l}
\mathrm{U}_{\text {met }}\left(\frac{\mathrm{d}_{\text {met }}}{\mathrm{H}_{\text {met }}}\right)^{\mathrm{a}_{\text {met }}} \cdot\left(\frac{\mathrm{h}}{\mathrm{d}}\right)^{\mathrm{a}} \rightarrow \mathrm{h}<\mathrm{d} \\
\mathrm{U}_{\text {met }}\left(\frac{\mathrm{d}_{\text {met }}}{\mathrm{H}_{\text {met }}}\right)^{\mathrm{a}_{\text {met }}} \rightarrow \mathrm{h} \geq \mathrm{d}
\end{array}\right.
$$


where $U_{\text {met }}$ is the wind speed measured from a nearby meteorological station, $\mathrm{H}_{\text {met }}$ is the anemometer height, $\mathrm{a}_{\text {met }}$ is the terrain factor for the meteorological station, $\mathrm{d}_{\text {met }}$ is the boundary layer thickness at the meteorological station, $a$ is the terrain factor for desired location, $d$ is the boundary layer thickness at desired location.

Moving vehicles intensify both micro and large scale mixing processes in the environment, by inducing turbulence and enhancing advection, by entraining masses of air in the direction of the vehicle motion [11]. So traffic is taken into account in the modelling with a moving layer below the object "car" that is a block used to simulate the vehicle.

Different wind speed scenarios were taken into account (Table 1).

Table 1: $\quad$ Meteorological input data.

\begin{tabular}{|l|c|c|c|c|c|c|c|}
\hline Simulation number & 1 & 2 & 3 & 4 & 5 & 6 & 7 \\
\hline Wind direction & East & East & East & S-E & S-E & S-E & South \\
\hline Wind velocity (m/s) & 1,0 & 2,5 & 4,0 & 1,0 & 2,5 & 4,0 & 4,0 \\
\hline
\end{tabular}

\section{Results and discussion}

\subsection{Measurements}

The CO concentration was measured by $\mathrm{CO}$ monitor based on the non-dispersive infrared method (NDIR) with a detection range of $0-50 \mathrm{ppm}$ and with detection limit of $0.5 \mathrm{ppm}$.

Fifteen-min average of 1 minute samples (reported at the end of each time period) of $\mathrm{CO}$ concentrations were measured in $\mathrm{n} .8$ different points within the street at the height of $3.00 \mathrm{~m}$, allowing an investigation of the spatial variability of the $\mathrm{CO}$ concentrations along a street canyon.

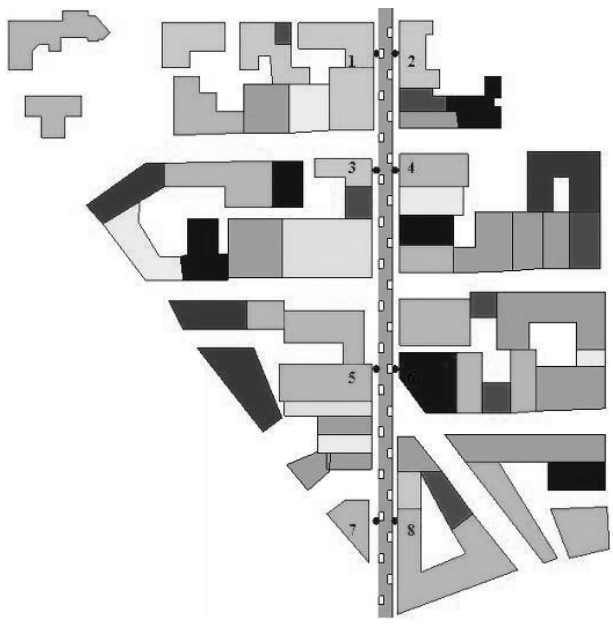

Figure 4: $\quad$ Points of measurement. 
The accuracy of the $\mathrm{CO}$ monitor sensor was verified by a calibration with a gas containing 5 ppm of $\mathrm{CO}$.

The turbulent mixing of the in-canyon volume of air, implies that any two measurements of a pollutant conducted at close proximity to each other in the street, can differ significantly.

The variables measured, in each point, were: $\mathrm{CO}$ maximum, minimum, average; wind speed, maximum, minimum, average; relative humidity, maximum, minimum; temperature, maximum, minimum, average.

In point 5 and $6, \mathrm{CO}$ concentration was measured at $3 \mathrm{~m}, 6 \mathrm{~m}$, and $9 \mathrm{~m}$ above ground.

The distribution of the $\mathrm{CO}$ concentration is not homogeneous in the street; the pollutant concentration is higher at the lower levels of the street than at the higher level. Moreover, in the lower part of the street we can observe a higher concentration on the leeward side than on the windward side of the street.

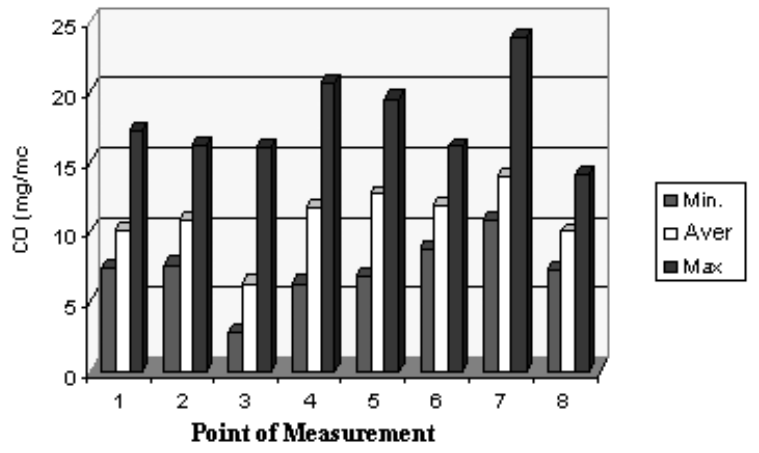

Figure 5: $\quad$ CO concentration of 12 January 2005 from 7:30 to 8:30.

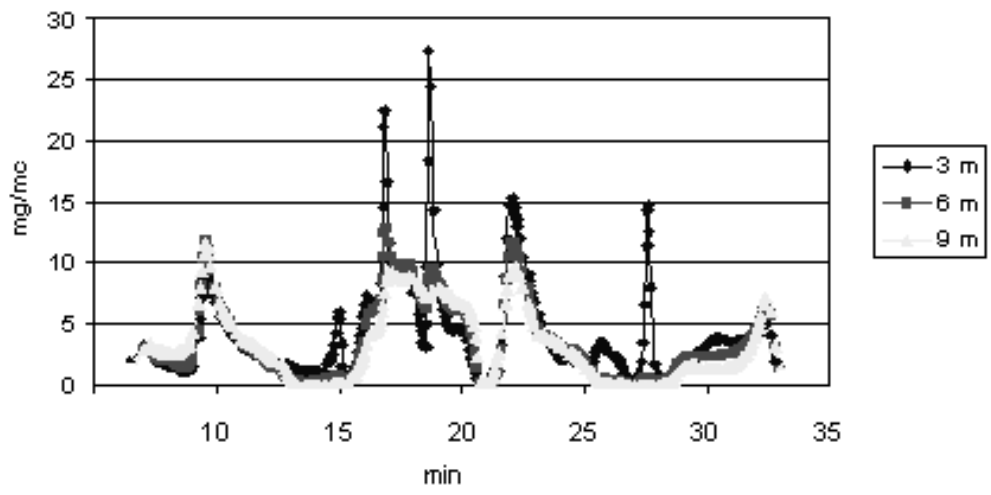

Figure 6: Evolution of $\mathrm{CO}$ concentrations in the windward side (point 6) during the 12 January 2005 from 08:00 to 8:30. The mean direction for the period was $82^{\circ}$ from the street axis and velocity $2.5 \mathrm{~m} / \mathrm{s}$. 


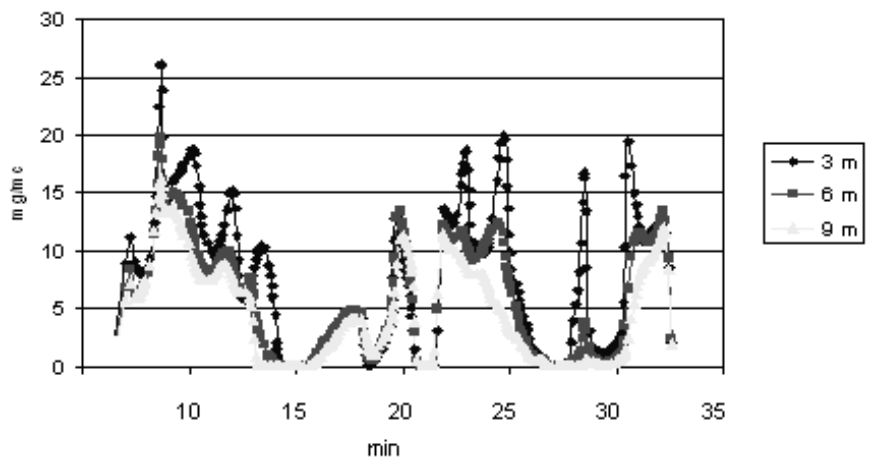

Figure 7: Evolution of CO concentrations in the leeward side (point 5) during the 12 January 2005 from 08:00 to 8:30. The mean direction for the period was $82^{\circ}$ from the street axis and velocity $2.5 \mathrm{~m} / \mathrm{s}$.

\subsection{Simulated velocity field and concentration}

In the case of direction of wind perpendicular to the canyon axis, the authors have noticed, according to the theoretical studies:

- The airflow in the canyon can be seen as a secondary circulation feature. If the wind speed out of the canyon is below $2,5 \mathrm{~m} / \mathrm{sec}$, the coupling between the upper and secondary flow is lost and the relation between the speed of the wind above the roof and within the canyon is characterized by a considerable scattering.

- Regarding the direction of the vortex, it has to be expected that, as the vortex is driven by a downward transfer of momentum across the rooflevel shear zone, the orthogonal flow to the canyon axis create a vortex with the air near the ground flowing opposite to the wind direction outside the canyon.
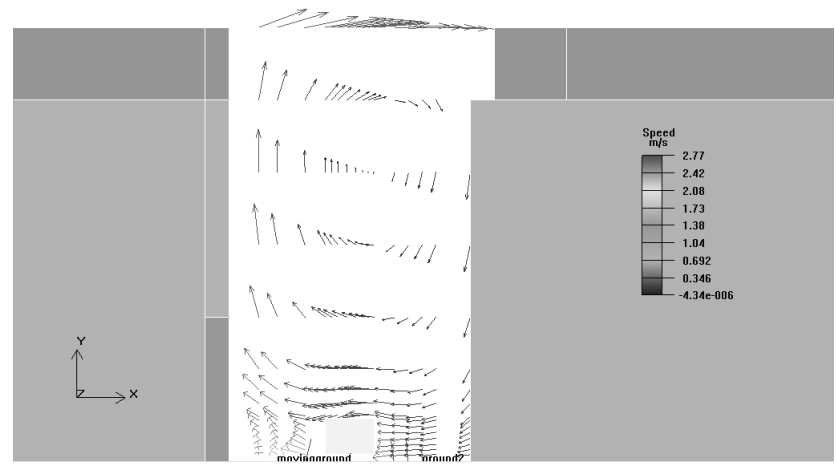

Figure 8: $\quad$ Flow pattern $-\mathrm{Pz}=60 \mathrm{~m}-\mathrm{Nz}=1-\mathrm{v}=1,0 \mathrm{~m} / \mathrm{sec} \perp$ Canyon. 


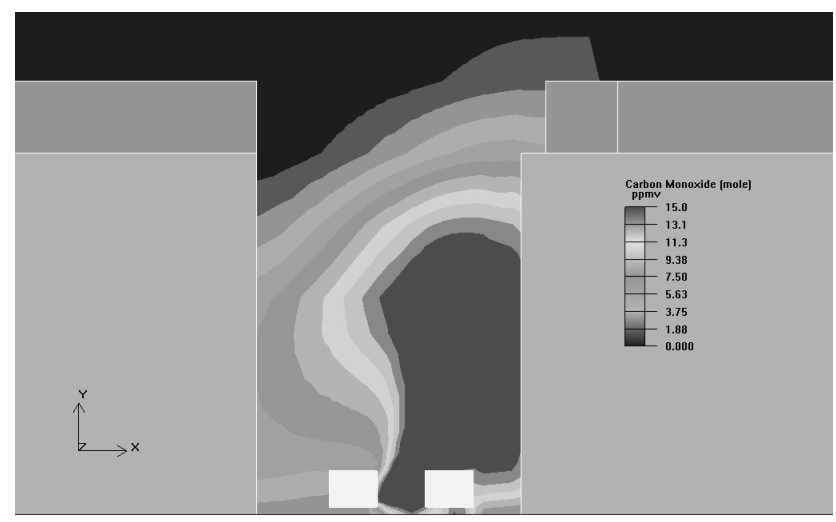

Figure 9: Exemplum of $\mathrm{CO}$ iso-concentration $\mathrm{Pz}=60 \mathrm{~m}-\mathrm{Nz}=1-$ $\mathrm{v}=1,0 \mathrm{~m} / \mathrm{sec} \perp$ canyon.

- The authors have found that high wind speeds $(>2,5 \mathrm{~m} / \mathrm{sec})$ produce transverse and longitudinal stable whirls between buildings façades.

- The transverse stable whirls are originated by the impact of vertical component of the wind on the windward building façades while the longitudinal stable whirls are originated both by the buildings with different heights, flanking the street, and by transversal streets converging in the canyon, which create vortex circulation.

- In this case the effects of the finite canyon length play an important role on the airflow distribution in the canyon. As a matter of fact the Authors have found intermittent vortices in the buildings corner. These vortices are responsible for the mechanism of advection from the building corner to the mid block creating a convergence zone in the mid block region of the canyon.

- Vertical velocities in the centre of the vortex of the canyon have been measured to be close to zero.

Moreover, the authors have noticed:

- An evident variation of the direction of velocity near the intersection with transversal streets that allow the inlet of air flow coming from zones outside the canyon.

- The biggest value of pollutants concentration forecasted close to the face of leeward buildings near the jets positions.

- The dispersions of pollutant gases inside the canyon very small both in longitudinal and in cross directions. As far as longitudinal direction is concerned, this is due to a very negligible component of wind vector, whereas, in the cross direction, the airflow motion forces the pollution between the facades of the building on both side.

- A very little component of wind velocity along the axis of canyon.

- An accumulation of polluting concentrations on leeward side of the building caused by buildings geometry, vortex circulation, transversal street and courtyards that supports the formation of depression zones. 


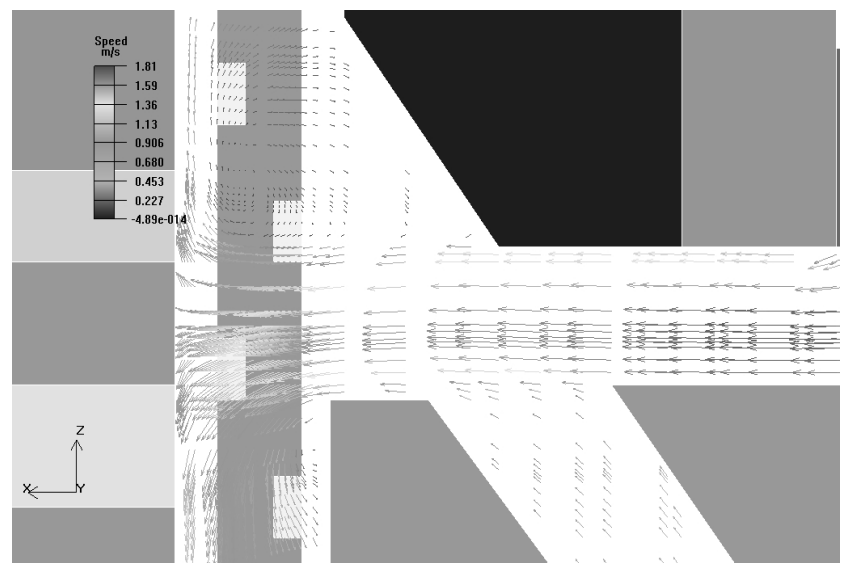

Figure 10: Exemplum of flow pattern $-\mathrm{Py}=3,00 \mathrm{~m}-\mathrm{Ny}=1-\mathrm{v}=2,5 \mathrm{~m} / \mathrm{sec}$.

- Values of concentrations between 510 and $50 \mathrm{mg} / \mathrm{m}^{3}$ of CO.

- The concentration of CO for leeward façades is twice as high as for the windward façades.

\subsection{Comparison between 3D simulations and measurements}

Both the above plots reveal that the predicted values generally follow the hourly trend of the measurements, related mainly to variations in traffic flow rate, and the pollutant concentrations on the leeward sides are higher than those on the windward sides. Whatever, the differences of average values of $\mathrm{CO}$ concentrations between simulations and measurements are 10-15\%. However, the predictions are quite close to the field measurements, although there are some differences the differences may follow from unsteadiness and large fluctuations, which, related to either traffic or approaching wind in actual environments, were not considered in the quasi-steady-state model.

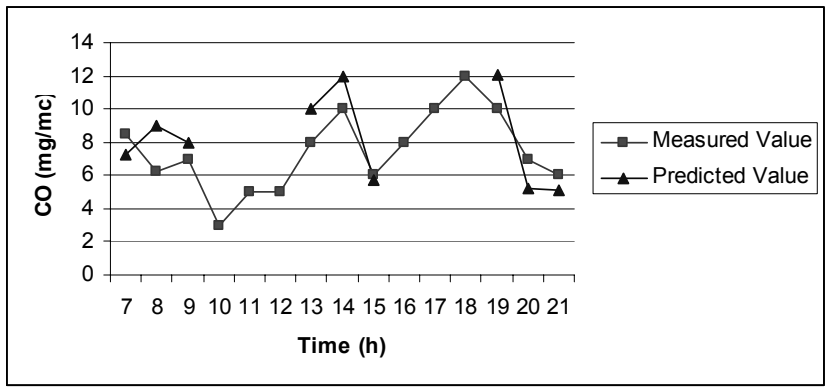

Figure 11: Comparisons between simulated and measured $\mathrm{CO}$ concentrations at a sampling height of $3.00 \mathrm{~m}$ in the windward side on 20 January 2005 from 07:00 to 21:00- the mean direction for the period was $85^{\circ}$ from the street axis and velocity $2.1 \mathrm{~m} / \mathrm{s}$. 


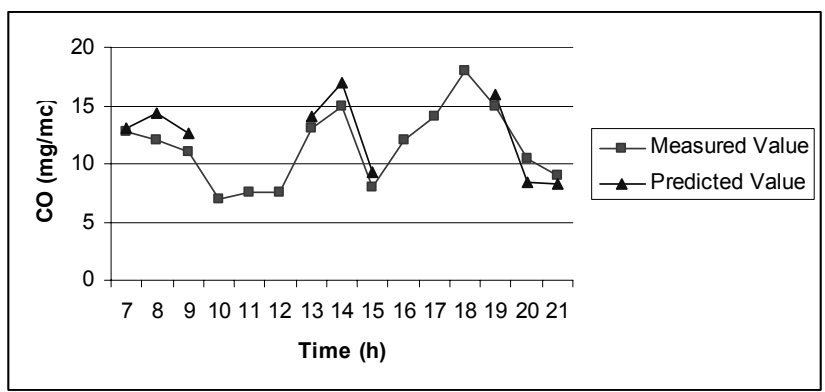

Figure 12: Comparisons between simulated and measured $\mathrm{CO}$ concentrations at a sampling height of $3.00 \mathrm{~m}$ in the leeward side on 20 January 2005 from 07:00 to 21:00 - the mean direction for the period was $85^{\circ}$ from the street axis and velocity $2.1 \mathrm{~m} / \mathrm{s}$.

\section{Conclusions}

The study shows that the model predictions of the spatial variability of any traffic-related pollutant in a canyon with adjoining side streets should be used to understand the complex flow regimes and turbulence structures that develop under certain background wind directions. This has special relevance to predicting the relative exposure of people to pollutants within street canyons.

However the experience obtained by this study shows that comparison of predicted and experimental results is difficult due to very unsteady wind conditions during field measurements. Experimental measurement are a necessary completion as a validation tool for predictions that has a very serious limitation due to unstable meteorological conditions that mostly prevail in a real situation.

\section{References}

[1] Croxford, B., Penn, A., Hillier, B., 1996. Spatial distribution of urban pollution: civilising urban traffic. Science of the Total Environment 189/190, 3-9.

[2] Sotiris Vardoulakis, Bernard E.A. Fisher, Koulis Pericleous, Norbert Gonzales-Flesca., 2003. Modelling air quality in street canyons: a review, Atmospheric Environment Vol 37, 155-182

[3] Nakamura, Y., Oke, T.R., 1988. Wind, temperature and stability conditions in an east-west oriented urban canyon. Atmospheric Environment 22, 2691-2700

[4] Oke, T.R., 1988. Street design and urban canopy lay and climate. Energy and Buildings 11, 103-113

[5] Hoydysh, W.G., Dabberdt, W.F., 1994. Concentration fields at urban intersections: fluid modelling studies. Atmospheric Environment 28 (11), 1849-1860. 
[6] Kastner-Klein, P., Plate, E.J., 1999. Wind-tunnel study of concentration fields in street canyons. Atmospheric Environment 33, 3973-3979.

[7] Zannetti, P., 1990. Air Pollution Modeling: Theories, Computational Methods, and Available Software. Computational Mechanics Publications, Southampton, UK

[8] Ntziachristos, L., Samaras, Z., 1997. COPERT II: Computer Programme to Calculate Emissions from Road Transport-User's Manual. European Topic Centre on Air Emissions, EEA, Copenhagen, Denmark.

[9] Ntziachristos, L., Samaras, Z., 2000. COPERT III: Computer Programme to Calculate Emissions from Road Transport-Methodology and Emission Factors. European Topic Centre on Air Emissions, EEA, Copenhagen, Denmark

[10] AIRPAK 2.1 User Guide Inc. 2001

[11] Xian-Xiang Li, Chun-Ho Liu, Dennis Y.C. Leung, K.M. Lam "Recent progress in CFD modelling of wind field and pollutant transport in street canyons" Atmospheric Environment 40 (2006) 5640-5658 